OPEN ACCESS

Edited by:

Bo Li,

Shandong University, China

Reviewed by:

Abhishek Kumar Srivastava, Indian Institute of Technology (BHU),

India

$\mathrm{Han} \mathrm{He,}$

National Astronomical Observatories

(CAS), China

*Correspondence:

Luis A. Balona

lab@saao.ac.za

Specialty section:

This article was submitted to

Stellar and Solar Physics,

a section of the journal

Frontiers in Astronomy and Space

Sciences

Received: 07 July 2020 Accepted: 23 February 2021

Published: 18 March 2021

Citation:

Balona LA (2021) Spots and Flares in Hot Main Sequence Stars Observed

by Kepler, K2, and TESS.

Front. Astron. Space Sci. 8:580907. doi: 10.3389/fspas.2021.580907

\section{Spots and Flares in Hot Main Sequence Stars Observed by Kepler, $K 2$, and TESS}

\author{
Luis A. Balona* \\ South African Astronomical Observatory, Cape Town, South Africa
}

About 22,000 Kepler stars, 7,000 K2 stars, and nearly 60,000 TESS stars from sectors 1-24 have been classified according to variability type. A large proportion of stars of all spectral types appear to have periods in their light curves consistent with the expected rotation periods. A previous analysis of A- and late B-type stars suggests that these stars are indeed rotational variables. In this paper we have accumulated data to show that rotational modulation is present in about $30-40 \%$ of A- and B-type stars. A search for flares in TESS A- and B-type stars resulted in the detection of 102 flares in 57 stars. Analysis of flare energies show that the source of the flares cannot be a cool dwarf companion nor a $\mathrm{F} / \mathrm{G}$ giant. The realization that a considerable fraction of A- and B-type stars are active indicates that a revision of current concepts regarding hot star envelopes is required.

Keywords: stellar activity, stellar rotation, star spots, flare stars, time series

\section{INTRODUCTION}

Spots on stars other than the Sun were first discovered by Kron (1947). Following the work of Hall (1972), it is now well-established that starspots similar to those in the Sun are present in stars with convective envelopes (Strassmeier, 2009). The presence of starspots leads to a brightness variation from which the rotation period can be found. Many thousands of solar-like stars with photometric rotational modulation have been detected from the Kepler space mission (McQuillan et al., 2013, 2014; Nielsen et al., 2013; Reinhold et al., 2013).

The rotational light modulation that is seen in cool stars with spots has never been detected in A- or B-type stars from ground-based photometry. However, high-precision space photometry of upper main sequence from the Kepler and $K 2$ missions show periodic or quasi-periodic light variations with periods consistent with the expected rotational periods of these stars (Balona, 2013, 2016, 2017). These studies were confined to A- and late B-type stars since only a small number of early B-type stars were observed by Kepler and K2. Balona (2019) extended the study to mid B-type stars using TESS Sectors 1-13. It appears that about 40\% of A-type stars (Balona, 2013) and about $30 \%$ of mid and late B-type stars (Balona, 2019) are rotational variables. However, from a much smaller sample, Sikora et al. (2020) estimates that the incidence of chemically normal rotational variables might be $<30 \%$. High-resolution spectroscopic time series of Vega (A0V) also indicates the presence of a spotted stellar surface (Böhm et al., 2015), providing independent confirmation of the photometric results. Savanov (2019) confirms the rotational character of the light variations in the A star, KIC 7047141. In this work we add further stars from TESS sectors 14-24, which now allow the rotational analysis to be extended to early B-type stars. Evidence for rotational light modulation among $\mathrm{O}$ stars has been presented by Buysschaert et al. (2015). 
Flares on the Sun and cool stars are thought to be a result of energy release through the magnetic reconnection process. It is therefore natural to associate the presence of starspots with flares since both arise from localized magnetic fields on the surface. Flares on $\mathrm{K}$ and $\mathrm{M}$ dwarfs are well documented since their discovery by Luyten (1949). The advent of the Kepler space mission (Borucki et al., 2010) led to the discovery of flares on several F- and A-type stars (Balona, 2012) and superflares on solar-type stars (Maehara et al., 2012). Further studies (Balona, 2013, 2015; Balona et al., 2016b) indicate that around 2.5\% of Atype stars flare with energies in the range $10^{35}-10^{36} \mathrm{erg}$. Švanda and Karlický (2016) find that of occurrence frequencies of stellar flares in A stars is systematically shifted toward a high-energy tail compared to stars of cooler spectral types. Pedersen et al. (2017) have argued that flares in A stars are likely a result of cool flare stars in the same aperture or binary companions. Direct evidence of possible X-ray flares in A-type stars have been reported by Schmitt et al. (1994) and Robrade and Schmitt (2010), while a flare on a B-type star has also been reported (Yanagida et al., 2004, 2007).

Rotational modulation and flares in A- and B-type stars implies the presence of surface magnetic fields. According to current views, magnetic fields in stars are either of fossil origin (i.e., the star was born with an embedded global magnetic field), or are generated by the dynamo effect in mass motions that occur in convective layers (Parker, 1955; Charbonneau, 2014). Fossil fields are used to account for the strong global magnetic fields in Ap and Bp stars (Mestel, 1967; Braithwaite and Spruit, 2004). Since the outer envelopes of A- and B-type stars lack the deep convective layers in the Sun and cool stars, it has long been assumed that most A- and B-type stars do not possess surface magnetic fields. However, recent improvements in technology have resulted in the detection of very weak global fields in some bright A-type stars such as Sirius and Vega (Petit et al., 2011) and on Am stars (Blazère et al., 2016a,b).

Stellar pulsation in upper main sequence stars also challenges our current concepts of the outer layers of A- and B-type stars. First results from the Kepler space mission on pulsations in mainsequence A-type stars (Grigahcène et al., 2010) already indicated a serious problem. It turns out that nearly all $\delta$ Scuti stars have multiple low frequency pulsations which cannot be explained by current models (Balona, 2014, 2018). A further surprise was the confirmation that many late B-type stars pulsate with high frequencies (Maia variables, Balona et al., 2015, 2016a; Balona and Ozuyar, 2020a). It does not seem possible to understand the pulsational instability of Maia variables as a consequence of incorrect opacities (Daszyńska-Daszkiewicz et al., 2017). Perhaps of even more significance is the fact that less than half of the stars in the $\delta$ Sct instability strip pulsate. Also, it seems that the $\gamma$ Dor variables may be just a subset of the $\delta$ Sct stars (Balona, 2018). None of these findings are explained by current pulsation models which may perhaps be a result of incorrect assumptions regarding the outer layers of A- and B-type stars.

New ideas regarding the outer layers of stars in radiative envelopes have recently emerged. It has been suggested, for example, that generation of magnetic fields by dynamo action may occur in sub-surface convective zones in A- and B-type stars (Cantiello et al., 2009; Cantiello and Braithwaite, 2011, 2019). At the surface they give rise to bright starspots. Also, it has been suggested that differential rotation may act to provide dynamo-generated magnetic fields in radiative zones (Tout and Pringle, 1995; Spruit, 1999, 2002; Maeder and Meynet, 2004). Whether or not these ideas may offer a solution to the detection of starspots and flares in A- and B-type stars, or help in resolving the challenges posed by pulsation, remains to be seen.

In this paper we report on further evidence for photometric rotational modulation among TESS A- and B-type stars, indicating that starspots are common among all B-type stars, including early B-type stars. We also report on a search for flares in TESS stars on the upper main sequence. On the basis of these results and the difficulty in explaining stellar pulsation in Aand B-type stars, we argue that current ideas regarding the outer layers of stars in radiative equilibrium need to be revised.

\section{DATA AND METHODOLOGY}

In searching for rotational modulation, the full 4-year light curves from long cadence $(30 \mathrm{~min})$ Kepler data, together with light curves from TESS ( 2 min cadence) sectors 1-24 were used. In both cases light curves using pre-search data conditioning (PDC) were selected (PDCSAP_SAP flux column in the FITS file). These are corrected for time-correlated instrumental signatures (Jenkins et al., 2016). In addition stars from the K2 mission (30 min cadence) were included.

In constructing the light curves, the mean was subtracted from each flux measurement and the result divided by the mean and multiplied by 1,000 to covert to parts-per-thousand (ppt). No corrections or rejection of data points were made and the data quality was not taken into account. Neither was there any attempt to smooth the data or adjust the zero points between quarters/sectors. The PDC data, exactly as given in the FITS files, were used. These are sufficient for identifying the rotation period which is typically only a few days and most often in the range $0.5-3 \mathrm{~d}$ in these rapidly-rotating stars. All stars with effective temperatures $T_{\text {eff }}>6,000 \mathrm{~K}$ brighter than magnitude 12.5 in the $V$ band were selected. There are 5,643 Kepler and K2 stars and over 50,000 TESS stars in this temperature range. As already mentioned, the data for the Kepler and K2 rotational variables have been described and published in Balona (2013, 2016, 2017). Many new TESS rotational variables were added to these in Balona (2019). The new TESS rotational variables added in this paper now include a sufficient number of early-B stars to show that rotational modulation is present even among the hottest B stars.

Visual inspection of the light curves and Lomb-Scargle periodograms (Scargle, 1982) of Kepler, K2, and TESS stars were used to assign variability types whenever appropriate. The variability classification follows that of the General Catalog of Variable Stars (GCVS, Samus et al., 2017). The only recognized class of rotational variables among the A- and B-type stars are the chemically peculiar $\alpha \mathrm{CVn}$ and SX Ari classes. A new ROT class has been added to describe any star which is not known to be Ap 
or Bp and in which the light variation is suspected to be a result of rotation.

The main indication of a ROT variable is a significant peak in the periodogram at a frequency which might correspond to the rotation frequency. A significant peak is one in which the amplitude exceeds the background noise by a factor of four or more. This significance criterion is justified by simulations as discussed in Koen (2010). In practice, the ROT classification is assigned to any single, isolated peak with a frequency less than about $4 \mathrm{~d}^{-1}$. Sometimes the first harmonic is also present, which adds additional credibility to the identification. When the harmonic is present, it is the fundamental which is taken to represent the rotation frequency. It is possible that in some stars the fundamental might be missing or have very low amplitude, in which case the period will half the true period. This might occur in stars with two diametrically located spots of about the same size. It is not possible to identify such cases from the available data. Periods longer than about $20 \mathrm{~d}$ are somewhat uncertain due to the increasing noise level in the periodogram.

The ROT type can be confused with low-level eclipses. In general, the ROT identification is confined to stars with amplitudes $<10$ millimagnitudes in an attempt to avoid this possibility. Aided by suitable software, visual classification of over 100 stars an hour is possible. In this way, several thousand stars with $T_{\text {eff }}>6,000 \mathrm{~K}$ have been assigned the ROT type.

The work on stellar flares is not meant to be a comprehensive survey of flares in TESS early-type stars. The flares reported here are those visible in both the corrected (PDCSAP_FLUX) and uncorrected (SAP_FLUX) light curves without removing the underlying stellar variation (detrending). No doubt, many more such flares remain to be discovered. Only the TESS data are discussed because flaring in Kepler early-type stars has already been reported (Balona, 2012, 2013, 2015; Balona et al., 2016b). For this purpose, the selection was limited to about 14,500 TESS stars with $T_{\text {eff }}>7,500 \mathrm{~K}$. This temperature was chosen in order to select stars likely to possess radiative envelopes.

It is particularly interesting to detect flares in A- and B-type stars since these would not be expected to occur, but would be a natural consequence of surface magnetic fields associated with the active regions in a starspot. For this purpose, we do not confine the search criterion to ROT stars only. In stars where the brightness is rapidly increasing or decreasing, it is sometimes difficult to detect flares unless they are of large amplitude. In such cases detrending is often necessary to render the flares more visible. For this reason, stars known to vary on a short timescale, such as the large number of $\delta$ Sct and other rapid variables with large amplitudes, were not included in the search for flares. This reduces the sample of 14,500 stars to 6,072 A- and 1,616 B-type stars.

\section{STELLAR PARAMETERS}

The most commonly used test for rotational modulation is comparison of the rotation rate derived from the photometric frequency, $v_{\mathrm{ROT}}$, with that derived from the projected rotational velocity, $v \sin i$. To derive the equatorial rotational velocity, $v$, from $v_{\text {ROT }}$ requires an estimate of the stellar radius, $R$. This can be done if we know the effective temperature, $T_{\text {eff }}$, and luminosity, $L / L_{\odot}$.

A catalog of over 101,500 stars comprising nearly 170,000 individual $T_{\text {eff }}$ measurements using various methods was compiled. For this purpose, the PASTEL catalogs (Soubiran et al., 2016) was very helpful, but many other references in the literature were searched. The most precise method of deriving $T_{\text {eff }}$ is by modeling absorption line profiles from medium- or highresolution spectroscopy. For A- and B-type stars, this involves fitting the $\mathrm{H} \beta$ and/or $\mathrm{H} \alpha$ line profiles because the strengths of the Balmer lines are sensitive to effective temperature. By comparing the observed line profiles with those generated by a suitable model atmosphere, the value of $T_{\text {eff }}$ and $\log g$ can be directly measured. The resulting standard deviation in $T_{\text {eff }}$ ranges from about $100 \mathrm{~K}$ for A-type stars to about $1,000 \mathrm{~K}$ for early B stars. Spectroscopic estimates of $T_{\text {eff }}$ exist for about $25 \%$ of the sample considered here.

The next best method is the use of narrow-band photometry. This involves measuring the strength of the $\mathrm{H} \beta$ line (the Strömgren $\beta$ index) usually in conjunction with $u v b y$ narrowband photometry. The $\beta$ index is sensitive to $T_{\text {eff }}$, but a color index is necessary to distinguish between A- and B-type stars. The value of $T_{\text {eff }}$ is obtained either by direct comparison with synthetic photometry derived from model atmospheres or by using stars with known $T_{\text {eff }}$ (Moon and Dworetsky, 1985; Gray, 1991; Napiwotzki et al., 1993; Smalley and Dworetsky, 1993; Balona, 1994). Estimates of $T_{\text {eff }}$ from Sloan ugriz (Brown et al., 2011) are of this type and are available for most of the Kepler stars. However, they cannot be used for stars earlier than A0 because they lack $u$-band measurements. Without the $u$ band, it is impossible to distinguish between A- and B-type stars of the same color. The same problem occurs with the majority of TESS effective temperatures for B-type stars. However, when these were in approximate agreement with $T_{\text {eff }}$ estimated from the spectral type, the TESS value was used. Estimates of $T_{\text {eff }}$ using narrowand intermediate-band photometry are available for about 55\% of stars.

If neither spectroscopy or narrow-band photometry is available, wide-band photometry can be used to estimate $T_{\text {eff }}$ provided that the reddening is known. This method is used in $7 \%$ of our sample. Finally, if nothing else is available, a crude estimate of $T_{\text {eff }}$ can be derived from the spectral classification together with suitable calibration such as the calibration of Pecaut and Mamajek (2013). This method was used for $18 \%$ of the stars.

The stellar luminosity is best estimated from Gaia DR2 parallaxes (Gaia Collaboration et al., 2016, 2018) in conjunction with reddening derived from a three-dimensional map by Gontcharov (2017) and the bolometric correction calibration by Pecaut and Mamajek (2013). From the error in the Gaia DR2 parallax, the typical standard deviation in $\log \left(L / L_{\odot}\right)$ is estimated to be about $0.05 \mathrm{dex}$, allowing for standard deviations of $0.01 \mathrm{mag}$ in the apparent magnitude, $0.10 \mathrm{mag}$ in visual extinction and $0.02 \mathrm{mag}$ in the bolometric correction in addition to the parallax error.

Table 1 lists the number of stars classified as ROT variables in the given range of effective temperature. Also shown is the 
TABLE 1 | Number of ROT stars within the given $T_{\text {eff }}$ range, $N_{\text {ROT }}$.

\begin{tabular}{lccc}
\hline $\boldsymbol{T}_{\text {eff }}$ & $\boldsymbol{N}_{\text {ROT }}$ & $\boldsymbol{f}_{\text {ROT }}$ & $\boldsymbol{N}_{\text {vsini }}$ \\
\hline $6,000-7,000$ & 21835 & 0.50 & 3329 \\
$7,000-8,000$ & 3298 & 0.34 & 239 \\
$8,000-10,000$ & 2418 & 0.31 & 420 \\
$10,000-12,000$ & 529 & 0.40 & 205 \\
$12,000-18,000$ & 341 & 0.37 & 219 \\
$18,000-30,000$ & 138 & 0.29 & 85 \\
\hline
\end{tabular}

Also shown is the fraction of ROT stars, $f_{R O T}$ within the range. $N_{v s i n i}$ is the number of stars used to construct the $v \sin i$ vs $v$ diagrams (Figure 1).

fraction of main sequence stars for which the ROT classification was assigned. Rotational modulation occurs in about $30-40 \%$ of A- and B-type stars. Note that Be stars were excluded from the sample. While the light variations in Be stars can be interpreted as rotational modulation, the light amplitude is typically an order of magnitude larger than for non-Be stars (Balona and Ozuyar, 2020b). It is suggested that the cause of the variability are co-rotating clouds which obscure a larger fraction of the photosphere than starspots. Because of the large amplitude, Be stars are disproportionately represented among the ROT stars. Since Be stars are rapid rotators, their inclusion leads to an over-estimate of the proportion of ROT stars with rapid rotation. Most Be stars are of early B type and this leads to a severe distortion of the velocity distribution for stars with $T_{\text {eff }}>18,000 \mathrm{~K}$.

\section{ROTATIONAL MODULATION}

The photometric period, obtained from Kepler/K2 and TESS light curves together with the stellar radius is used to estimate the equatorial rotational velocity, $v$. If the variability is rotational modulation, there should be a relationship between $v$ and the projected rotational velocity, $v \sin i$. It is expected that the rotational axes are randomly orientated. In this case the probability of the rotational axis being inclined at angle $i$ is proportional to $\sin i$. There will be a diminishing scatter of points below the $\sin i=1$ line due to stars with lower angles of inclination. Due to unavoidable errors (e.g., uncertainties in $v \sin i$, in the interstellar reddening, in the bolometric correction, in the parallax, and in the effective temperature), the resulting error in the stellar radius and the resulting equatorial rotational velocity is large, and it is to be expected that some points will lie above the $v \sin i=1$ line.

A catalog of projected rotational velocities, $v \sin i$ consisting of over 58,000 individual measurements of 35,200 stars was compiled. The bulk of these measurements are from Głębocki and Gnaciński (2005). The catalog was brought up to date by a literature search. The typical error in $v \sin i$ can be estimated from the catalog of Głębocki and Gnaciński (2005). The error increases with $v \sin i$ and ranges between 0 and $60 \mathrm{~km} \mathrm{~s}^{-1}$. A representative value of $\sigma_{v \sin i}=30 \mathrm{~km} \mathrm{~s}^{-1}$ is reasonable. From the error in $\log L / L_{\odot}$ and $T_{\text {eff }}$ it is easy to calculate the error in $v$. This error depends almost entirely on the error in $T_{\text {eff. }}$. The contribution from the luminosity error is small while the contribution from

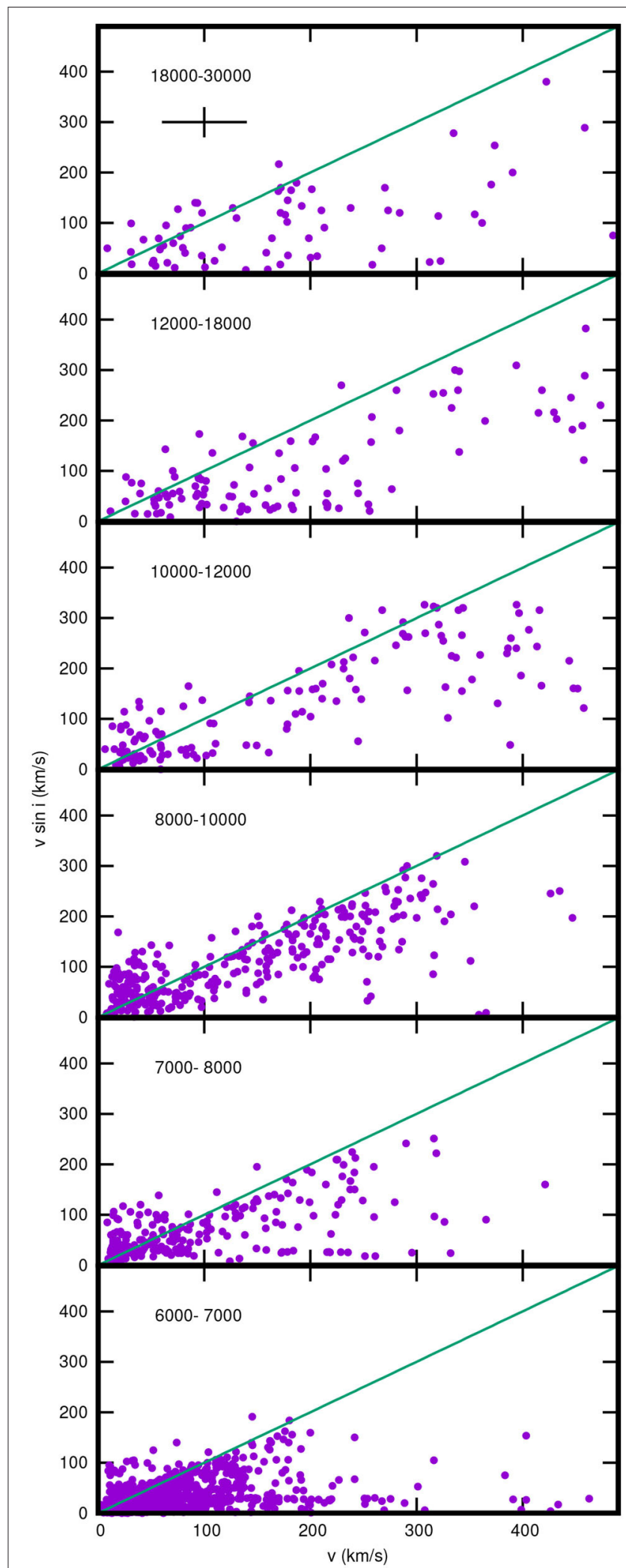

FIGURE 1 | The relationship between the projected rotational velocity, $v \sin i$ and the equatorial rotational velocity, $v$ (estimated from the photometric frequency), for different ranges of $T_{\text {eff }}$ (labeled). The cross in the top left panel shows the estimated 1- $\sigma$ error bars. The straight line corresponds to $v=v \sin i$. Be stars have been omitted. 
the error in the rotation period is entirely negligible. The typical value for the error in the derived equatorial rotational velocity is $\sigma_{v} \approx 40 \mathrm{~km} \mathrm{~s}^{-1}$.

Projected rotational velocities are not available for every star for which $v$ has been estimated. In Figure 1, the $v-$ $v \sin i$ diagram is shown for all stars for which measurements are available. The data used to construct this figure are available as Supplementary Material. For completeness, Kepler, $K 2$, and TESS stars used in previous analyses and with available $v \sin i$ measurements are included. There are many previously unpublished stars with photometric periods, but without $v \sin i$ measurements. These will be published elsewhere.

The typical error bars discussed above are indicated in the top left panel. It is clear that the expected distribution of points is present from the F- to the early B-type stars. This justifies the original assumption that the periodic light variation is due to rotation.

As expected, nearly all stars with $v>60 \mathrm{~km} \mathrm{~s}^{-1}$ lie on or below the $\sin i=1$ line. For low rotation rates, ever increasing observational precision is required to determine whether or not $v \sin i<v$. Often, $v \sin i$ values are truncated at some positive number corresponding to the resolution limit of the instrument. It becomes increasingly difficult to distinguish between binarity and rotation at low frequencies. For example, amplitude variability, which is a typical attribute of rotational modulation, is not so easily detected. Thus one may expect significant contamination from binaries at low rotation rates. These factors are probably responsible for the increased scatter in this region.

\section{TESS EARLY-TYPE FLARE STARS}

In our catalog of nearly 60,000 TESS stars classified for variability, there are 14,495 stars with $T_{\text {eff }}>7,500 \mathrm{~K}$. As already mentioned, eclipsing binaries, $\delta$ Sct stars and other types of rapid variables with high amplitudes require detrending to render the flares visible. To avoid this complication, only non-varying or slowlyvarying stars were selected in the search for flares. This results in 6,072 A- and 1,616 B-type stars in which the variability is not too rapid. Flares were detected by visual inspection of the light curves. The intention is not to detect every incidence of flares among the A- and B-type stars, but merely to show that such flares exist, as they do in the Kepler data.

A flare is taken to be an event consisting of a sharp rise and gradual fall in brightness. Flares of short duration (a few minutes) will be missed because the TESS observations have a cadence of $2 \mathrm{~min}$. It is possible, of course, that an event similar to a flare would be generated by a passing object (satellite or minor planet). Indeed, some examples of such events are visible in the TESS light curves, but their shapes are different from what might be expected in a flare. If events looking like typical flares are present in many stars, it becomes less likely that these are generated by passing objects. In particular, if these events occur several times in the same star (as found in a few stars), the probability that these are extrinsic to the star is negligible. If the events are due to some instrumental problem, then they should occur at the same time on a given CCD chip or possibly on the entire ensemble of chips, depending on the problem. No instance of two or more flares occurring at the same time was found. Nevertheless, it cannot be excluded that some events identified as flares may be due to other causes.

The 57 stars in Table 2 appear to have at least one flare-like event. In this table the stellar parameters are shown. A substantial proportion of the flare stars are X-ray sources. Table 3 gives information on all flare-like events that were detected. The rise time, $t_{\text {rise }}$ is the time taken for the flare to reach maximum intensity, while $t_{\text {decay }}$ is the time from maximum intensity to the time when the intensity reaches $1 / \mathrm{e}$ of its maximum value (the efolding time). In deriving these values, the flare background was removed by fitting a polynomial of low degree or, in some cases, by fitting a spline curve.

Examples of flares are shown in Figure 2. Multiple flares are visible in 21 stars, giving a total of 102 flare events. Not included here are the TESS A-type flare stars TIC 118327563 and TIC 224244458 (Balona et al., 2019a). The former is a sdB star and the latter is an SX Ari variable (Bp star). Also excluded is the $\delta$ Sct flare star TIC 439399707 (Balona et al., 2019b) and the Be X-ray source TIC 207176480 (Balona and Ozuyar, 2020b).

The number of TESS A-type stars which appear to flare constitute about $1 \%$ of the sample of A-type stars which were examined, which is less than half of the $2.5 \%$ flare incidence among Kepler A-type stars reported by Balona (2013, 2015). This can be understood given the fact that the long-cadence Kepler data span 4 years, while the TESS data mostly span a few months and always less than one year. There are 61 Kepler A-type stars known to flare (Balona, 2015). The additional 57 flare stars reported here do not include any of the Kepler stars, bringing the total of flaring A-type stars to 118.

Because the stellar continuum is very weak in the UV, flare amplitudes in cool dwarfs increase very rapidly toward the UV. The TESS photometric pass band is 6,000-11,000 $\AA$ where flare emission is much lower and the stellar continuum much higher. This means that cool star flare amplitudes observed by TESS are very much lower compared with observations in the blue and UV. Any cool star in the aperture would need to be of comparable brightness to the A star for the flare to be detected. Whereas, the continuum of a cool star in the near infra-red is relatively brighter than that of an A star of the same visual magnitude, the flare amplitude will be lower. Therefore, the flare visibility would probably not be greatly affected whether the relative magnitude difference is measured in the visual or in the near infra-red.

Whereas the Kepler pixel size is 4 arcsec, the TESS pixel size is 21 arcsec. This means that the probability of a flare originating in a star other than the A star is much larger for TESS. The stars are all very bright (4.2-10.4 mag), with most in the range 6-8 mag. The fields of all 57 stars were examined using the 2MASS, DSS images in SIMBAD. In every case the A star is by far the dominant optical source and no companion of significant brightness was found within a radius of $1 \mathrm{arcmin}$. The Gaia database (Gaia Collaboration et al., 2016, 2018) was examined for stars within a radius of 1 arcmin, but none brighter than about 12.5 mag was found. TIC 256749693 and TIC 360020620 are close doubles with magnitude differences of 3.2 and 1.7 mag respectively. 
TABLE 2 | Flare stars detected from visual inspection of TESS light curves.

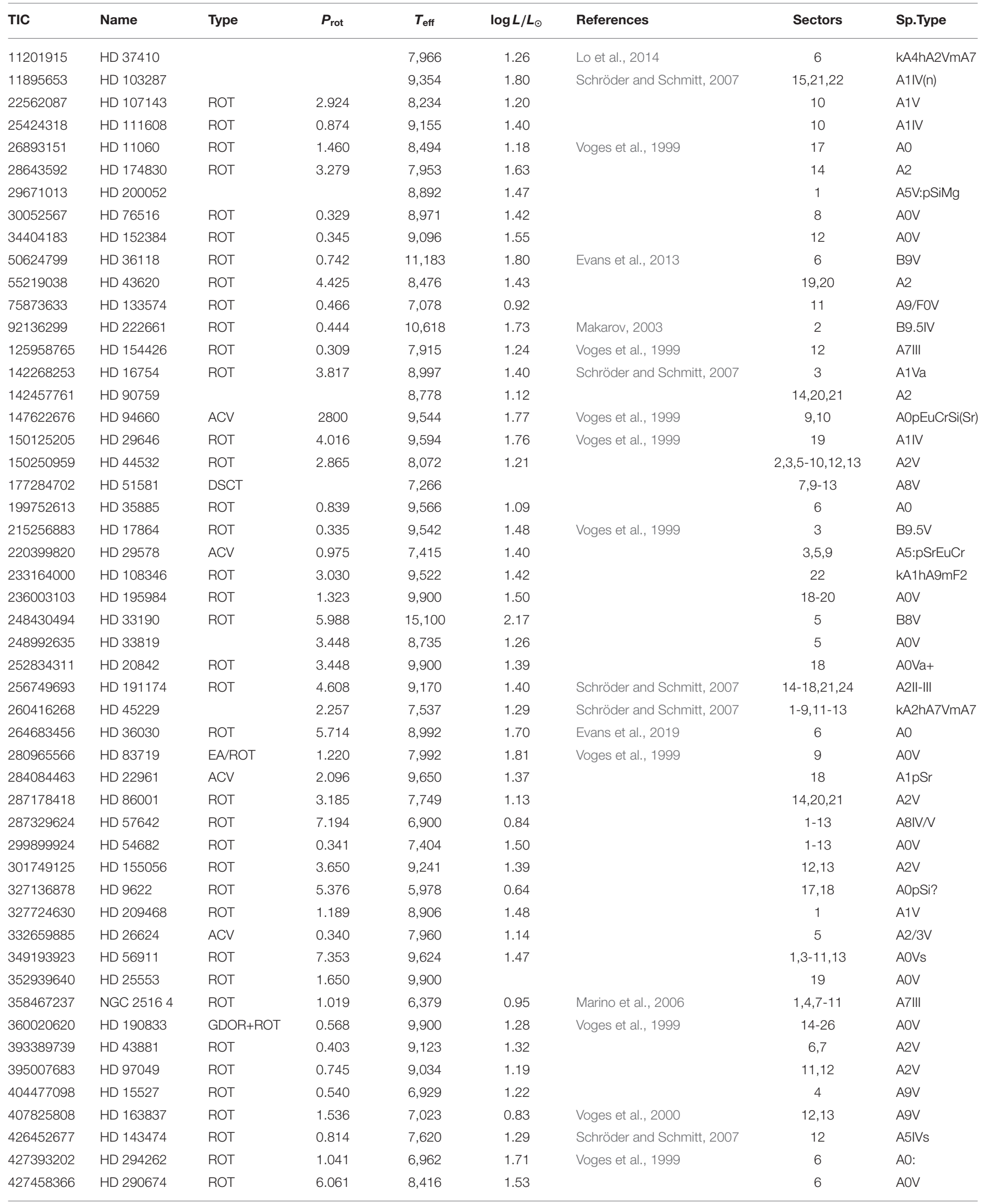




\section{TABLE 2 | Continued}

\begin{tabular}{|c|c|c|c|c|c|c|c|c|}
\hline TIC & Name & Type & $P_{\text {rot }}$ & $T_{\text {eff }}$ & $\log L / L_{\odot}$ & References & Sectors & Sp.Type \\
\hline 438598966 & HD 116649 & ROT & 1.055 & 8,200 & 1.22 & & 11 & AOV \\
\hline 440863421 & HD 131885 & ROT & 3.636 & 8,793 & 1.47 & & 11 & AOV \\
\hline 443316662 & HD 45341 & ROT & 5.076 & 7,159 & 1.13 & & 6 & $\mathrm{~A} 2$ \\
\hline 452468734 & HD 80950 & ROT & 16.129 & 10,069 & 1.61 & & 10-12 & AOV \\
\hline
\end{tabular}

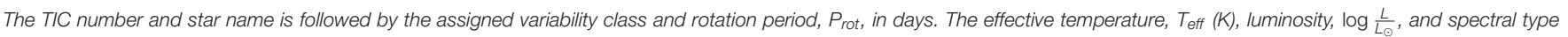
are also shown. Many stars appear to be X-ray sources as given by the literature reference. Finally, the spectral type is shown.

A search for foreground cool dwarfs was made using the catalog of Muirhead et al. (2018) which lists over 1 million of these stars. Only three A-type stars were found where a cool dwarf was present within a radius of $2 \mathrm{arcmin}$. None of these cool dwarfs was brighter than $14.4 \mathrm{mag}$ (the minimum magnitude difference being $6.5 \mathrm{mag}$ ). It is thus very unlikely that a cool dwarf is the source of the flare in any of the A-type stars. Therefore, the flares must be due to exotic faint objects, a physical companion of the A star or the A star itself.

The flare energy was estimated by integration of the flare light curve. From the estimated stellar luminosity the energy radiated by the flare can then be determined. This assumes that the wavelength distribution of flux from the flare is about the same as from the star. If the flux distribution in the flare corresponds to a blackbody with temperature higher than about $10,000 \mathrm{~K}$, the true energies will be larger. Thus, Schmitt et al. (2019) adopt a blackbody flux distribution corresponding to 12,000 K, while Froning et al. (2019) and Howard et al. (2020) use $40,000 \mathrm{~K}$. From Table 3 the range in flare energy is $10^{33.5}$ to $10^{36} \mathrm{erg}$ with a mean of $10^{34.5} \mathrm{erg}$, but these may be just lower limits.

\section{CONCLUSION AND DISCUSSION}

It is demonstrated that the periodic light variations seen in about $30-40 \%$ of A- and B-type stars is consistent with rotational modulation. The expected relationship between the estimated equatorial rotation velocity, $v$, and the projected rotation velocity, $v \sin i$, is confirmed in several effective temperature ranges.

The nature of the presumed starspots responsible for rotational modulation is not known. The idea of magnetic field generation in subsurface convective zones first postulated by Cantiello et al. $(2009,2011)$ and Cantiello and Braithwaite (2011, 2019) seems to be quite promising. According to Cantiello and Braithwaite (2019), the largest effects are caused by a convective layer driven by second helium ionization. The amplitude of surface magnetic fields and their associated photometric variability are expected to decrease with increasing stellar mass and surface temperature, so that magnetic spots and their observational effects should be much harder to detect in late Btype stars. This is clearly not the case, since the fraction of late B-type stars showing rotational modulation is about the same as in A-type stars (Table 1). In fact, the fraction stays about the same at $30-40 \%$ for all stars in the upper main sequence.

Another problem is that sub-surface convection predicts the creation of bright spots. We know that spots on the Sun are dark, and this seems to be true of solar-type stars as well. Since rotational modulation is present for the full range of main sequence stars, there must be a transition between dark spots and bright spots around early F or late $\mathrm{A}$. As a result, one might expect a decrease in the numbers of stars with rotational modulation in this spectral type range. This does not seem to be the case unless the transition is very sharp.

An alternative mechanism proposed many years ago involves the interaction between magnetic fields, convective flows and differential rotation. A dynamo cycle operating on differential rotation in stellar radiative interiors was described by Tout and Pringle (1995), Spruit (1999, 2002), and Maeder and Meynet (2004) (see also Braithwaite and Spruit 2017). In this theory, a magnetic instability in the toroidal field wound up by differential rotation replaces the role of convection in closing the field amplification loop in conventional dynamo theory. It is possible that completely stable radiative envelopes do not exist and that turbulence generated by differential rotation may lead to surface magnetic fields capable of forming conventional dark spots.

Examination of TESS A- and B-type stars has led to the detection of 57 new flare stars in addition to the 61 early-type flare stars already known from the Kepler mission. These new flare stars include some Ap and Am stars, with some stars being $\mathrm{X}$-ray sources. If starspots are deemed to be present, in A- and Btype stars, then there should be no barrier to accepting that flares may be generated by magnetic reconnection, as in the Sun and cool stars. Indeed, it then becomes necessary to provide reasons why flares should not be generated in A- and B-type stars.

Cool dwarfs are plentiful, of course, but extremely faint. The apparent magnitudes of the TESS A-type stars are 4-10 mag with a median of $8.2 \mathrm{mag}$. A cool dwarf of comparable brightness, or even significantly fainter, will be one of the nearest stars and welldocumented. Using a catalog of over one million cool dwarfs (Muirhead et al., 2018), it is found that a foreground cool dwarf within a radius of 2 arcmin cannot be the cause of the flares in A-type stars, which is not surprising. If the flare does not originate in a cool dwarf or the A star, then it must originate in an exotic faint object in the field or a physical companion to the A star. 
TABLE 3 | List of individual flares for stars in Table 2.

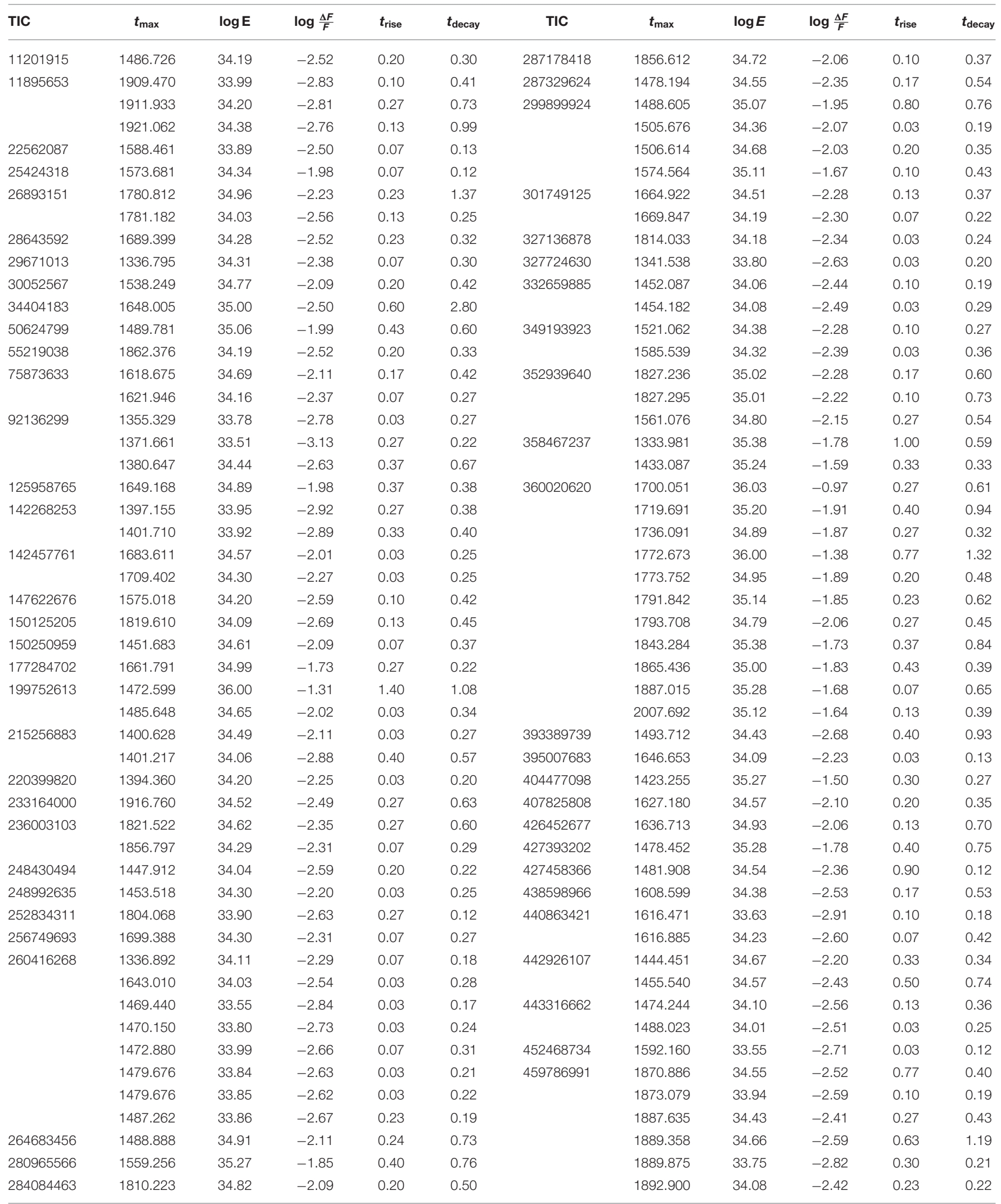

The time of peak flare intensity, $t_{\max }$, is relative to BJD 2457000. The flare energy, $E$ (erg), and the logarithm of relative peak flare intensity, log $\frac{\Delta F}{F}$ is shown. The flare rise time, $t_{\text {rise, }}$ and the e-fold decay time, $t_{\text {decay }}$, are in hours. 

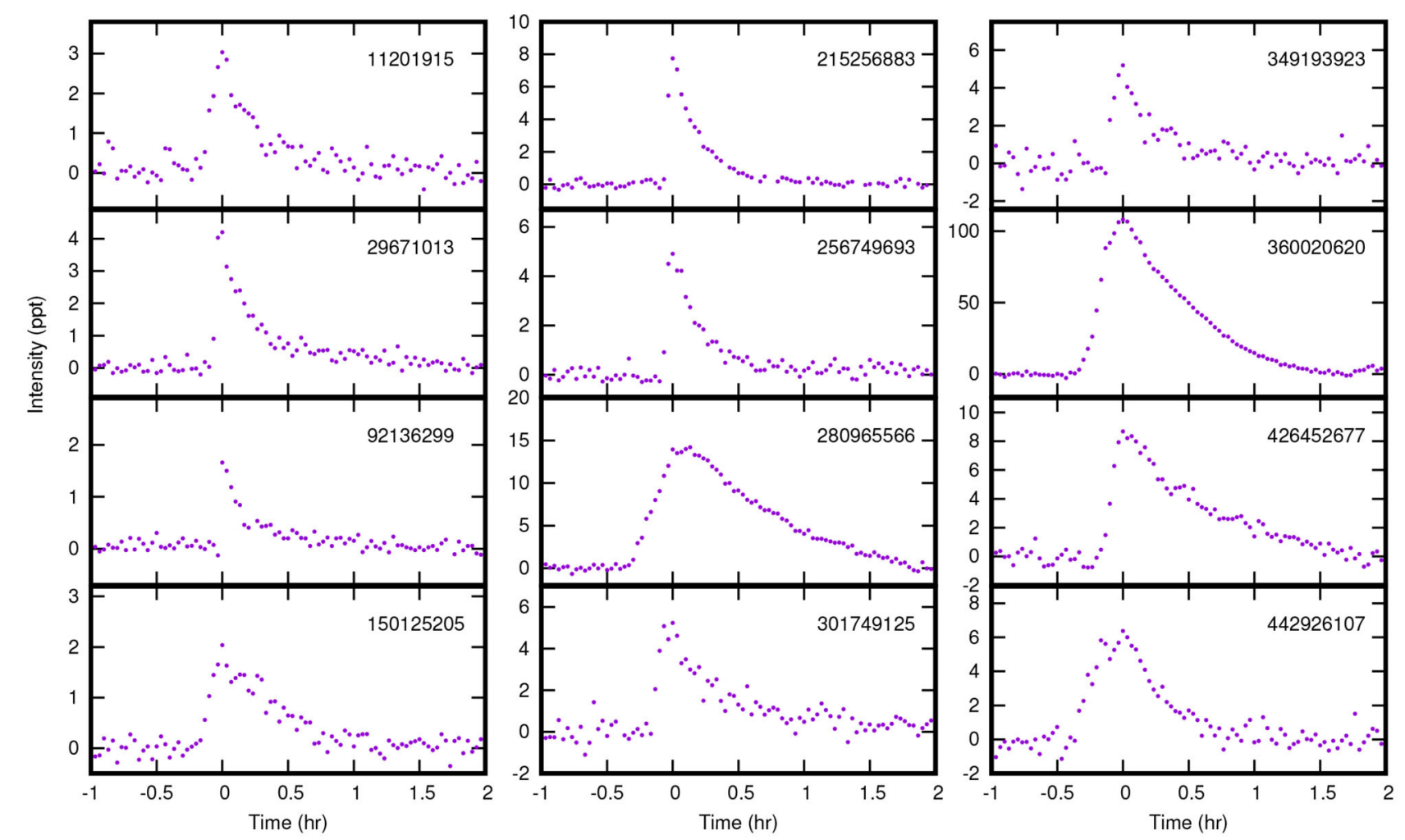

FIGURE 2 | Examples of A star flares in the TESS data. The time origin is the time of maximum flare intensity. Time is in hours and intensity if parts per thousand.

As can be seen from Table 3, a typical A-star flare energy is about $10^{34}-10^{35} \mathrm{erg}$. These could be lower energy limits. While it is true that flares in cool dwarfs may attain such high energies on rare occasions, the question that needs to be asked is how many cool stars with flares in this energy range are likely to be observed by TESS within the same time interval. From nearly 25,000 cool stars observed by TESS, only a single M-type star out of 1,228 flare stars was observed with a bolometric flare energy as high as $10^{34.7} \mathrm{erg}$ (Günther et al., 2020). This does not, of course, imply that flares of even higher energy cannot occur in cool dwarfs. However, it does give an indication of the rarity of superflares in these stars. What needs to be examined is the probability of finding superflares in cool-star companions to 57 A-type stars with the observed energies in the same time interval and observed in the same way.

To answer this question, from the numbers presented by Günther et al. (2020), it can be deduced that superflares occur in about $0.004 \%$ of cool dwarfs observed in the first 2 months of the TESS mission. Most TESS stars are observed for only one or two sectors, so this is roughly comparable to the time span per star for the A- and B-type stars discussed here. Over this time interval, superflares are found in $1 \%$ of TESS A-type stars. This is much higher than the expected $0.004 \%$ and does not even include the multiple superflares in A-type stars which occur within several days, something which has never been observed among cool flare stars. It seems that a physical cool star companion can be excluded as the source of the flares.

Superflares among F- and G-type giants have been discovered (Balona, 2012; Maehara et al., 2012). However, the A-type stars in which flares have been detected (Table 2) are bright and wellobserved. Spectroscopic observations give mostly early A types with no indication of F- or G-type giant companions. Since such companions would have luminosities similar to the A-type star, they should have been easily detected during spectroscopic classification. They would also have modified the photometric effective temperature estimates to cooler values, which is not the case. There is no evidence to suppose that flares on A-type stars originate in luminous F- or G-type physical companions either.

The above arguments show that a cool star or an F/G giant can be excluded as the source of flaring in A-type stars. There are two remaining possibilities: the flare arises in magnetic reconnection involving the A star and a close companion, or solely on the A star itself. Either way it means that a significant magnetic field must be present on the A star. This is, of course, what the rotational modulation observations suggest. We therefore return to the original problem regarding the presence of magnetic fields in radiative envelopes.

For further progress it will be important to design observations which might lead to resolving the problem of whether the spots are bright or dark. Further high-resolution spectroscopy of A- or B-type stars, as performed by Böhm et al. 
(2015) on Vega, would be important to place limits on the size and distribution of the spots. It would also be important to obtain time-series spectroscopy on A- and B-type flare stars to determine possible interacting companions.

Above all, work on revising our existing notions of the physics in the outer layers of stars with radiative envelopes needs to be conducted. New ideas on how magnetic fields may be generated in such stars are required. The unexpected discoveries in pulsations in A- and B-type stars provide important clues. Perhaps a useful starting point is to determine what processes are required to generate low-frequency pulsations in $\delta$ Scuti stars or the enigmatic high frequencies in the B-type pulsating Maia variables.

\section{DATA AVAILABILITY STATEMENT}

The raw data supporting the conclusions of this article will be made available by the authors, without undue reservation.

\section{AUTHOR CONTRIBUTIONS}

The author confirms being the sole contributor of this work and has approved it for publication.

\section{FUNDING}

Funding for the TESS mission was provided by the NASA Explorer Program. Funding for the TESS Asteroseismic Science

\section{REFERENCES}

Balona, L. A. (1994). Effective temperature bolometric correction and mass calibration of O-F. Mon. Not. R. Astron. Soc. 268:119-127. doi: 10.1093/mnras/268.1.119

Balona, L. A. (2012). Kepler observations of flaring in A-F type stars. Mon. Not. R. Astron. Soc. 423, 3420-3429. doi: 10.1111/j.1365-2966.2012.21135.x

Balona, L. A. (2013). Activity in A-type stars. Mon. Not. R. Astron. Soc. 431, 2240-2252. doi: 10.1093/mnras/stt322

Balona, L. A. (2014). Low frequencies in Kepler $\delta$ Scuti stars. Mon. Not. R. Astron. Soc. 437, 1476-1484. doi: 10.1093/mnras/stt1981

Balona, L. A. (2015). Flare stars across the H-R diagram. Mon. Not. R. Astron. Soc. 447, 2714-2725. doi: 10.1093/mnras/stu2651

Balona, L. A. (2016). Rotational modulation in B stars observed by the Kepler K2 mission. Mon. Not. R. Astron. Soc. 457, 3724-3731. doi: 10.1093/mnras/stw244

Balona, L. A. (2017). Starspots on A stars. Mon. Not. R. Astron. Soc. 467, 1830-1837. doi: $10.1093 / \mathrm{mnras} / \mathrm{stx} 265$

Balona, L. A. (2018). Gaia luminosities of pulsating A-F stars in the Kepler field. Mon. Not. R. Astron. Soc. 479, 183-191. doi: 10.1093/mnras/sty1511

Balona, L. A. (2019). Evidence for spots on hot stars suggests major revision of stellar physics. Mon. Not. R. Astron. Soc. 490, 2112-2116. doi: $10.1093 / \mathrm{mnras} / \mathrm{stz} 2808$

Balona, L. A., Baran, A. S., Daszyńska-Daszkiewicz, J., and De Cat, P. (2015). Analysis of Kepler B stars: rotational modulation and Maia variables. Mon. Not. R. Astron. Soc. 451, 1445-1459. doi: 10.1093/mnras/stv1017

Balona, L. A., Engelbrecht, C. A., Joshi, Y. C., Joshi, S., Sharma, K., Semenko, E., et al. (2016a). The hot $\gamma$ Doradus and Maia stars. Mon. Not. R. Astron. Soc. 460, 1318-1327. doi: 10.1093/mnras/stw1038

Balona, L. A., Handler, G., Chowdhury, S., Ozuyar, D., Engelbrecht, C. A., Mirouh, G. M., et al. (2019a). Rotational modulation in TESS B stars. Mon. Not. R. Astron. Soc. 485, 3457-3469. doi: 10.1093/mnras/stz586
Operations Centre was provided by the Danish National Research Foundation (Grant agreement no.: DNRF106), ESA PRODEX (PEA 4000119301) and Stellar Astrophysics Centre (SAC) at Aarhus University. This work has made use of data from the European Space Agency (ESA) mission Gaia, processed by the Gaia Data Processing and Analysis Consortium (DPAC). Funding for the DPAC has been provided by national institutions, in particular the institutions participating in the Gaia Multilateral Agreement. This research has made use of the SIMBAD database, operated at CDS, Strasbourg, France. Data were obtained from the Mikulski Archive for Space Telescopes (MAST). STScI was operated by the Association of Universities for Research in Astronomy, Inc., under NASA contract NAS5-2655.

\section{ACKNOWLEDGMENTS}

I wish to thank the National Research Foundation of South Africa for financial support. I also thank the TESS Asteroseismic Science Operations Center (TASOC).

\section{SUPPLEMENTARY MATERIAL}

The Supplementary Material for this article can be found online at: https://www.frontiersin.org/articles/10.3389/fspas. 2021.580907/full\#supplementary-material
Balona, L. A., Holdsworth, D. L., and Cunha, M. S. (2019b). High frequencies in TESS A-F main-sequence stars. Mon. Not. R. Astron. Soc. 487, 2117-2132. doi: $10.1093 / \mathrm{mnras} / \mathrm{stz} 1423$

Balona, L. A., and Ozuyar, D. (2020a). Pulsation among TESS A and B stars and the Maia variables. Mon. Not. R. Astron. Soc. 493, 5871-5879. doi: 10.1093/mnras/staa670

Balona, L. A., and Ozuyar, D. (2020b). TESS observations of Be stars: a new interpretation. Mon. Not. R. Astron. Soc. 493, 2528-2544. doi: 10.1093/mnras/staa389

Balona, L. A., Švanda, M., and Karlický, M. (2016b). Differential rotation, flares and coronae in A to M stars. Mon. Not. R. Astron. Soc. 463, 1740-1750. doi: $10.1093 / \mathrm{mnras} / \mathrm{stw} 2109$

Blazère, A., Neiner, C., and Petit, P. (2016a). Discovery of a very weak magnetic field on the Am star Alhena. Mon. Not. R. Astron. Soc. 459, L81-L84. doi: 10.1093/mnrasl/slw050

Blazère, A., Petit, P., Lignières, F., Aurière, M., Ballot, J., Böhm, T., et al. (2016b) Detection of ultra-weak magnetic fields in Am stars: $\beta$ Ursae Majoris and $\theta$ Leonis. Astron. Astrophys. J. 586:A97. doi: 10.1051/0004-6361/201527556

Böhm, T., Holschneider, M., Lignières, F., Petit, P., Rainer, M., Paletou, F., et al. (2015). Discovery of starspots on Vega. First spectroscopic detection of surface structures on a normal A-type star. Astron. Astrophys. J. 577:A64. doi: 10.1051/0004-6361/201425425

Borucki, W. J., Koch, D., Basri, G., Batalha, N., Brown, T., Caldwell, D., et al. (2010). Kepler planet-detection mission: introduction and first results. Science 327:977-980.

Braithwaite, J., and Spruit, H. C. (2004). A fossil origin for the magnetic field in A stars and white dwarfs. Nature 431, 819-821. doi: 10.1038/nature02934

Braithwaite, J., and Spruit, H. C. (2017). Magnetic fields in non-convective regions of stars. R. Soc. Open Sci. 4:160271. doi: 10.1098/rsos.160271

Brown, T. M., Latham, D. W., Everett, M. E., and Esquerdo, G. A. (2011). Kepler input catalog: photometric calibration and 
stellar classification. Astron. J. 142:112. doi: 10.1088/0004-6256/ $142 / 4 / 112$

Buysschaert, B., Aerts, C., Bloemen, S., Debosscher, J., Neiner, C., Briquet, M., et al. (2015). Kepler's first view of O-star variability: K2 data of five O stars in Campaign 0 as a proof of concept for O-star asteroseismology. Mon. Not. R. Astron. Soc. 453, 89-100. doi: 10.1093/mnras/stv1572

Cantiello, M., and Braithwaite, J. (2011). Magnetic spots on hot massive stars. Astron. Astrophys. 534:A140. doi: 10.1051/0004-6361/201117512

Cantiello, M., and Braithwaite, J. (2019). Envelope convection, surface magnetism, and spots in A and late B-type stars. Astrophys. J. 883:106. doi: 10.3847/1538-4357/ab3924

Cantiello, M., Braithwaite, J., Brandenburg, A., Del Sordo, F., Käpylä, P., and Langer, N. (2011). "3D MHD simulations of subsurface convection in OB stars," in Active OB Stars: Structure, Evolution, Mass Loss, and Critical Limits, Volume 272 of IAU Symposium, eds C. Neiner, G. Wade, G. Meynet, and G.Peters (Cambridge: Cambridge University Press), 32-37.

Cantiello, M., Langer, N., Brott, I., de Koter, A., Shore, S. N., Vink, J. S., et al. (2009). Sub-surface convection zones in hot massive stars and their observable consequences. Astron. Astrophys. 499, 279-290. doi: 10.1051/0004-6361/200911643

Charbonneau, P. (2014). Solar dynamo theory. Annu. Rev. Astron. Astrophys. 52, 251-290. doi: 10.1146/annurev-astro-081913-040012

Daszyńska-Daszkiewicz, J., Walczak, P., and Pamyatnykh, A. (2017). "On possible explanations of pulsations in Maia stars," in European Physical Journal Web of Conferences, Vol. 160 of European Physical Journal Web of Conferences, 03013.

Evans, P. A., Osborne, J. P., Beardmore, A. P., Page, K. L., Willingale, R., Mountford, C. J., et al. (2013). VizieR Online Data Catalog: 1SXPS Swift XRay Telescope Point Source Catalog (Evans+ 2014). VizieR Online Data Catalog, IX/43.

Evans, P. A., Page, K. L., Osborne, J. P., Beardmore, A. P., Willingale, R., Burrows, D. N., et al. (2019). VizieR Online Data Catalog: 2SXPS Swift X-Ray Telescope Point Source Catalog (Evans+, 2020). VizieR Online Data Catalog, IX/58.

Froning, C. S., Kowalski, A., France, K., Loyd, R. O. P., Schneider, P. C., Youngblood, A., et al. (2019). A hot ultraviolet flare on the M Dwarf star GJ 674. Astrophys. J. Lett. 871:L26. doi: 10.3847/2041-8213/aaffcd

Gaia Collaboration, Brown, A. G. A., Vallenari, A., Prusti, T., de Bruijne, J. H. J., Babusiaux, C., et al. (2018). Gaia Data Release 2. Summary of the contents and survey properties. ArXiv e-prints. doi: 10.1051/0004-6361/201833051

Gaia Collaboration, Prusti, T., de Bruijne, J. H. J., Brown, A. G. A., Vallenari, A., Babusiaux, C., et al. (2016). The Gaia mission. Astron. Astrophys. 595:A1. doi: $10.1051 / 006-6361 / 201629272$

Głębocki, R., and Gnaciński, P. (2005). "Systematic errors in the determination of stellar rotational velocities," in 13th Cambridge Workshop on Cool Stars, Stellar Systems and the Sun, Vol. 560 of ESA Special Publication, eds F. Favata, G. A. J. Hussain, and B. Battrick, 571.

Gontcharov, G. A. (2017). 3D stellar reddening map from 2MASS photometry: an improved version. Astron. Lett. 43, 472-488. doi: 10.1134/S1063773717070039

Gray, R. O. (1991). The calibration of Stromgren photometry for A, F and early G supergiants.II. The F and early G supergiants. Astron. Astrophys. 252:237.

Grigahcène, A., Antoci, V., Balona, L., Catanzaro, G., Daszyńska-Daszkiewicz, J., Guzik, J. A., et al. (2010). Hybrid $\gamma$ Doradus- $\delta$ scuti pulsators: new insights into the physics of the oscillations from Kepler observations. Astrophys. J. Lett. 713, L192-L197. doi: 10.1088/2041-8205/713/2/L192

Günther, M. N., Zhan, Z., Seager, S., Rimmer, P. B., Ranjan, S., Stassun, K. G., et al. (2020). Stellar flares from the first TESS data release: exploring a new sample of M dwarfs. Astrophys. J. 159:60. doi: 10.3847/1538-3881/ab5d3a

Hall, D. S. (1972). A T Tauri-like star in the eclipsing binary RS canum venaticorum. Publ. Astron. Soc. Pac. 84:323. doi: 10.1086/129291

Howard, W. S., Corbett, H., Law, N. M., Ratzloff, J. K., Galliher, N., Glazier, A. L., et al. (2020). EvryFlare. III. Temperature evolution and habitability impacts of dozens of superflares observed simultaneously by evryscope and TESS. Astrophys. J. 902:115. doi: 10.3847/1538-4357/abb5b4

Jenkins, J. M., Twicken, J. D., McCauliff, S., Campbell, J., Sanderfer, D., Lung, D., et al. (2016). Jenkins, J. M., Twicken, J. D., McCauliff, S., Campbell, J., Sanderfer, D., Lung, D., et al. (2016). "The TESS science processing operations center," in Software and Cyberinfrastructure for Astronomy IV, Volume 9913 of Proceedings SPIE, eds G. Chiozzi, and J. C. Guzman, 99133E.
Koen, C. (2010). The "four sigma" limit for significant periodicities. Astrophys. Space Sci. 329, 267-271. doi: 10.1007/s10509-009-0199-z

Kron, G. E. (1947). The probable detecting of surface spots on AR lacertae B. Publ. Astron. Soc. Pac. 59:261. doi: 10.1086/125964

Lo, K. K., Farrell, S., Murphy, T., and Gaensler, B. M. (2014). Automatic classification of time-variable X-ray sources. Astrophys. J. 786:20. doi: 10.1088/0004-637X/786/1/20

Luyten, W. J. (1949). A new star of large proper motion (L 726-8). Astrophys. J. 109:532. doi: $10.1086 / 145158$

Maeder, A., and Meynet, G. (2004). Stellar evolution with rotation and magnetic fields. II. General equations for the transport by Tayler-Spruit dynamo. Astron. Astrophys. 422, 225-237. doi: 10.1051/0004-6361:20034583

Maehara, H., Shibayama, T., Notsu, S., Notsu, Y., Nagao, T., Kusaba, S., et al. (2012). Superflares on solar-type stars. Nature 485, 478-481. doi: $10.1038 /$ nature 11063

Makarov, V. V. (2003). The 100 brightest X-ray stars within 50 parsecs of the sun. Astron. J. 126, 1996-2008. doi: 10.1086/378164

Marino, A., Micela, G., Pillitteri, I., and Peres, G. (2006). X-ray variability of NGC 2516 stars in the XMM-Newton observations. Astron. Astrophys. 456, 977-983. doi: 10.1051/0004-6361:20054674

McQuillan, A., Mazeh, T., and Aigrain, S. (2013). Stellar rotation periods of the Kepler objects of interest: a dearth of close-in planets around fast rotators. Astrophys. J. Lett. 775:L11. doi: 10.1088/2041-8205/775/1/L11

McQuillan, A., Mazeh, T., and Aigrain, S. (2014). Rotation periods of 34,030 Kepler main-sequence stars: the full autocorrelation sample. Astrophys. J. Suppl. Ser. 211:24. doi: 10.1088/0067-0049/211/2/24

Mestel, L. (1967). Mestel, L. (1967). "Fossil' magnetic fields and the oblique-rotator model," in Magnetic and Related Stars, ed R. C. Cameron (Baltimore, MD: Mono Book Corporation), 101.

Moon, T. T., and Dworetsky, M. M. (1985). Grids for the determination of effective temperature and surface gravity of B, A and F stars using uvby-beta photometry. Mon. Not. R. Astron. Soc. 217, 305-315. doi: 10.1093/mnras/217.2.305

Muirhead, P. S., Dressing, C. D., Mann, A. W., Rojas-Ayala, B., Lépine, S., Paegert, M., et al. (2018). A catalog of cool dwarf targets for the transiting exoplanet survey satellite. Astron. J. 155:180. doi: 10.3847/1538-3881/aab710

Napiwotzki, R., Schoenberner, D., and Wenske, V. (1993). On the determination of effective temperature and surface gravity of B, A, and F stars using Stromgren UVBY beta photometry. Astron. Astrophys. 268, 653-666.

Nielsen, M. B., Gizon, L., Schunker, H., and Karoff, C. (2013). Rotation periods of 12000 main-sequence Kepler stars: dependence on stellar spectral type and comparison with V sin I observations. Astron. Astrophys. 557:L10. doi: 10.1051/0004-6361/201321912

Parker, E. N. (1955). Hydromagnetic dynamo models. Astrophys. J. 122:293. doi: $10.1086 / 146087$

Pecaut, M. J., and Mamajek, E. E. (2013). Intrinsic colors, temperatures, and bolometric corrections of pre-main-sequence stars. Astrophys. J. Suppl. Ser. 208:9. doi: 10.1088/0067-0049/208/1/9

Pedersen, M. G., Antoci, V., Korhonen, H., White, T. R., Jessen-Hansen, J., Lehtinen, J., et al. (2017). Do A-type stars flare? Mon. Not. R. Astron. Soc. 466, 3060-3076. doi: 10.1093/mnras/stw3226

Petit, P., Lignières, F., Wade, G. A., Aurière, M., Alina, D., Böhm, T., et al. (2011). Weak magnetic fields of intermediate-mass stars. Astron. Nachr. 332:943. doi: 10.1002/asna.201111608

Reinhold, T., Reiners, A., and Basri, G. (2013). Rotation and differential rotation of active Kepler stars. Astron. Astrophys. 560:A4. doi: 10.1051/0004-6361/201321970

Robrade, J., and Schmitt, J. H. M. M. (2010). X-ray emission from the remarkable A-type star HR 8799. Astron. Astrophys. 516:A38. doi: $10.1051 / 0004-6361 / 201014027$

Samus, N. N., Kazarovets, E. V., Durlevich, O. V., Kireeva, N. N., and Pastukhova, E. N. (2017). General catalog of variable stars: Version GCVS 5.1. Astron. Rep. 61, 80-88. doi: 10.1134/S1063772917010085

Savanov, I. S. (2019). "Activity of A-type star KIC 7047141," in Physics of Magnetic Stars, Vol. 518 of Astronomical Society of the Pacific Conference Series, eds D. O. Kudryavtsev, I. I. Romanyuk, and I. A. Yakunin, 195. 
Scargle, J. D. (1982). Studies in astronomical time series analysis. II - Statistical aspects of spectral analysis of unevenly spaced data. Astrophys. J. 263, 835-853. doi: $10.1086 / 160554$

Schmitt, J. H. M. M., Guedel, M., and Predehl, P. (1994). Spatially resolved X-ray and radio observations of Castor A+B+C. Astron. Astrophys. 287, 843-851.

Schmitt, J. H. M. M., Ioannidis, P., Robrade, J., Czesla, S., and Schneider, P. C. (2019). Superflares on AB Doradus observed with TESS. Astron. Astrophys. 628:A79. doi: 10.1051/0004-6361/201935374

Schröder, C., and Schmitt, J. H. M. M. (2007). X-ray emission from A-type stars. Astron. Astrophys. 475, 677-684. doi: 10.1051/0004-6361:20077429

Sikora, J., Wade, G. A., and Rowe, J. (2020). A spectroscopic test of the rotational modulation origin of periodic Kepler photometric variability of A-type stars. Mon. Not. R. Astron. Soc. 498, 2456-2471. doi: 10.1093/mnras/staa2444

Smalley, B., and Dworetsky, M. M. (1993). The atmospheric parameters of A-stars and F-stars - part one - comparison of various methods. Astron. Astrophys. 271:515-526.

Soubiran, C., Le Campion, J.-F., Brouillet, N., and Chemin, L. (2016). The PASTEL catalog: 2016 version. Astron. Astrophys. 591:A118. doi: 10.1051/0004-6361/201628497

Spruit, H. C. (1999). Differential rotation and magnetic fields in stellar interiors. Astron. Astrophys. 349, 189-202.

Spruit, H. C. (2002). Dynamo action by differential rotation in a stably stratified stellar interior. Astron. Astrophys. 381, 923-932. doi: 10.1051/0004-6361:20011465

Strassmeier, K. G. (2009). Starspots. Astron. Astrophys. Rev. 17, 251-308. doi: 10.1007/s00159-009-0020-6

Švanda, M., and Karlický, M. (2016). Flares on A-type stars: evidence for heating of solar corona by nanoflares? Astrophys. J. 831:9. doi: 10.3847/0004-637X/831/1/9
Tout, C. A., and Pringle, J. E. (1995). X-ray coronae from dynamos in young Ae/Be stars. Mon. Not. R. Astron. Soc. 272, 528-530. doi: 10.1093/mnras/272.3.528

Voges, W., Aschenbach, B., Boller, T., Bräuninger, H., Briel, U., Burkert, W., et al. (1999). The ROSAT all-sky survey bright source catalog. Astron. Astrophys. 349, 389-405.

Voges, W., Aschenbach, B., Boller, T., Brauninger, H., Briel, U., Burkert, W., et al. (2000). Rosat all-sky survey faint source catalog. iaucirc 7432:3.

Yanagida, T., Ezoe, Y., Kawaharada, M., Kokubun, M., and Makishima, K. (2007). "Large X-ray flares from B-type stars, HD261902 and HD47777, in NGC2264 observed with CHANDRA," in Active OB-Stars: Laboratories for Stellare and Circumstellar Physics, Vol. 361 of Astronomical Society of the Pacific Conference Series, eds A. T. Okazaki, S. P. Owocki, and S. Stefl, 533.

Yanagida, T., Ezoe, Y.-I., and Makishima, K. (2004). X-Ray flares from a late B-type star, HD 38563S, in NGC 2068 observed with chandra Xray observatory. Publ. Astron. Soc. Jpn. 56, 813-818. doi: 10.1093/pasj/ 56.5 .813

Conflict of Interest: The author declares that the research was conducted in the absence of any commercial or financial relationships that could be construed as a potential conflict of interest.

Copyright (C) 2021 Balona. This is an open-access article distributed under the terms of the Creative Commons Attribution License (CC BY). The use, distribution or reproduction in other forums is permitted, provided the original author $(s)$ and the copyright owner(s) are credited and that the original publication in this journal is cited, in accordance with accepted academic practice. No use, distribution or reproduction is permitted which does not comply with these terms. 